TUMOR CELL, MOLECULAR BIOLOGY, PREDICTIVE AND PROGNOSTIC FACTORS - OTHER

https://doi.org/10.29289/259453942021V31S1056

\title{
5-YEAR SURVIVAL ACCORDING TO THE MOLECULAR SUBTYPE OF 9,516 BREAST CANCER PATIENTS TREATED IN A REFERENCE CENTER
}

Nathália Karina Abreu¹, Bruno Neto Martins Aguiar ${ }^{1}$, André Mattar ${ }^{1}$, Jorge Yoshinori Shida ${ }^{1}$, Luiz Henrique Gebrim¹

${ }^{1}$ Hospital Pérola Byington - São Paulo (SP), Brazil.

Introduction: Breast cancer in Brazil has a high mortality rate due to the large number of patients diagnosed and treated in advanced stages, due to lack of an organized screening program and the long time from diagnosis to initiation of treatment in the Unified Health System (SUS). For the 2020-2022 triennium, the National Cancer Institute (INCA) estimated 66 thousand new cases of breast cancer among Brazilian women. In the city of São Paulo, 5,350 new cases were estimated, with a gross rate of 80.81/100,000. The biomarkers (RE, RP, Her2 and Ki-67) in breast carcinomas allowed for division into four subgroups: luminal A, luminal B, overexpression of HER2 and triple-negatives and greatly contributed to the specificity therapy with an impact on patient survival. The data about survival in SUS is scarce in the literature. Objectives: To assess 5-year survival in different molecular subtypes in 9,516 patients treated for breast cancer from January 2011 to December 2019 at Pérola Byington Hospital (PBH). Methods: The official database of PBH from January 2011 to December 2019 included 11,373 cases of breast cancer. Based on that, we proposed an observational, cross-sectional and retrospective study and we could include 9,516 patients treated for invasive breast cancer which had data available to analyze overall survival according to the molecular type of the tumor, regardless of the tumor clinical stage. Discussion: The luminal molecular subtype A (present in 24.7\%) had better survival (92\% in five years), the luminal subtype B was the most frequent, (32.8\%) and the slightly lower survival, $88 \%$. The ones that had the shortest survival, especially in the first two years, were the HER-2 subtypes present in $17.5 \%$ and the triple-negative ones (25.1\% of the patients). There are few data in Brazil evaluating the molecular subtypes and survival. The great number of triple-negative tumors is probably due to the fact that it was a population of untraceable women under the age of 50. Conclusions: The molecular subtypes of breast cancer are important to guide therapeutic measures and to predict the prognosis of patients. With data obtained at Hospital Pérola Byington, it can be concluded that the Luminal A subtypes are the ones with the best prognosis, while the Triple negative and Her 2 positive had higher mortality, especially over the first two years. 